\title{
Predominant global glomerulosclerosis in patients of upper urinary tract urothelial carcinoma with pre-existing renal function impairment is a predictor of poor renal outcomes
}

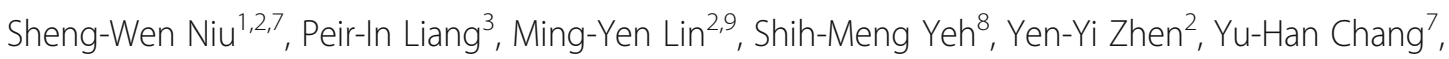 \\ Pin-Chia Huang ${ }^{7}$, Chi-Chi Hung ${ }^{2,6}$, I-Ching Kuo ${ }^{1,2,7}$, Hugo You-Hsien Lin' ${ }^{2,6}$, Mei-Chuan Kuo ${ }^{4,6}$, Wei-Ming Li, ${ }^{4,6}$, \\ Chun-Nung Huang ${ }^{4,6}$, Wen-Jeng Wu ${ }^{4,6,7}$, Li-Tzong Chen ${ }^{5}$, Yi-Wen Chiu ${ }^{2,6}$ and Shang-Jyh Hwang ${ }^{2,6^{*}}$
}

\begin{abstract}
Background: Incidence of renal dysfunction and risks of progression to end-stage renal disease (ESRD) were reported higher in upper urinary tract urothelial carcinoma (UTUC) than in renal cell carcinoma (RCC) patients after unilateral nephrectomy.

Methods: Totally 193 renal cancer patients, including 132 UTUC and 61 RCC, were studied to clarify whether the pathological changes of the kidney remnant removed from nephrectomy and the clinical factors might predict the risk of ESRD. Renal tubulointerstitial (TI) score and global glomerulosclerosis (GGS) rate were examined by one pathologist and two nephrologists independently under same histopathological criteria.

Results: The glomerular filtration rates at the time of surgery were lower in UTUC than RCC groups $(p<0.001)$. Average GGS score and average TI rate were higher in UTUC than in RCC groups $(p<0.001 ; p<0.001)$. Competitive risk factor analysis revealed that abnormal GGS rate not related to age, predominant in UTUC with pre-existing renal function impairment, was a histopathological predictor of poor renal outcomes (creatinine doubling or ESRD) within 5 years in UTUC patients.

Conclusion: Pre-existing renal function and pathological change of kidney remnant in both UTUC and RCC have the value for prediction of renal outcomes.

Keywords: Upper urinary tract urothelial carcinoma, Renal cell carcinoma, Renal survival, Tubulointerstitial nephropathy, Glomerulosclerosis
\end{abstract}

\section{Background}

The outcomes of renal cancers after surgical unilateral nephrectomy include patient survival and possibility of renal function deterioration to end-stage renal disease (ESRD). Pathology of renal cancers can be divided into renal

\footnotetext{
* Correspondence: sjhwang@kmu.edu.tw

${ }^{2}$ Division of Nephrology, Department of Internal Medicine, Kaohsiung

Medical University Hospital, Kaohsiung Medical University, No. 100, Tzyou 1st

Road, 80708 Kaohsiung, Taiwan

${ }^{6}$ Faculty of Medicine, Kaohsiung Medical University, Kaohsiung, Taiwan

Full list of author information is available at the end of the article
}

cell carcinoma (RCC) [1], origin from renal tubules, and urothelial cell carcinoma of renal pelvis and /or proximal ureter, named as upper urinary tract urothelial carcinoma (UTUC) [2]. RCC is much more common than UTUC in western countries [3], and UTUC contributes only $5 \%$ to all urothelial carcinoma (UC) [4]. However, prevalence of UTUC is not low in Taiwan, accounting for approximately $30 \%$ of all UCs [5], and is significantly, even 100 times higher, in areas endemic with aristolochic acid nephropathy (AAN) than the non-endemic counterparts [6].

(c) The Author(s). 2019 Open Access This article is distributed under the terms of the Creative Commons Attribution 4.0 International License (http://creativecommons.org/licenses/by/4.0/), which permits unrestricted use, distribution, and 
The status of renal function in UTUC patients after surgical intervention of unilateral nephrectomy could be of normal, or of various stages of chronic kidney disease (CKD), or progression to ESRD. Our previous study demonstrated that $10.7 \%$ of UTUC patients underwent dialysis treatment [7]. Pathological findings of AAN contained extensive chronic tubulointerstitial (TI) fibrosis but sparse of glomerulosclerosis [8], which also resulted in progression into ESRD. Furthermore, AAN was reported strongly associated with the development of UTUC [9], and high incidence of urothelial cancers (UTUC and bladder cancer) were noted in ESRD patients received either renal transplantation or dialysis [10].

Despite that the exact mechanism of AAN and UTUC was still not explored completely, we were interested in status of renal function in relation to the histopathological changes of the kidney remnant from nephrectomy in UTUC and RCC patients. Clinical factors and pathological parameters were analyzed for prediction of renal survival (creatinine doubling and ESRD) after unilateral nephrectomy in groups of UTUC and RCC patients.

\section{Methods}

\section{Subject data}

We retrospectively analyzed data of 132 non-metastatic UTUC patients between 2002 and 2010, and 61 non-metastatic RCC patients between 2003 and 2011. All patients had undergone unilateral nephrectomy through either open or laparoscopic approach at our hospitals. Parameters of age, sex, smoking, Chinese herb use, and prevalence of hypertension, diabetes mellitus, hyperlipidemia, hydronephrosis, and kidney stones were recorded. We excluded subjects who had incomplete clinical information, received renal replacement therapy preoperatively, had no pathological evidence of UTUC, and had undergone surgery twice for UTUC. Location of tumor defined as either ureter or renal pelvis based on dominant tumor features, in a sequential order of the stage, grade, and size. The renal histopathological parameters were investigated by 3 specialists: 2 nephrologists and a pathologist. Moreover, the subjects were stratified into quartiles according to age $(\leq 54,55-64$, 65-74, and $\geq 75$ years) and sex for pre-existing CKD prevalence and other analyses. The flow chart is described in Fig. 1. The study protocol was approved by our Institutional Review Board (KMUH-IRB-20120138).

\section{Pre-existing CKD evaluation}

To evaluate the patient's pre-operation kidney function, the latest creatinine level obtained within 30 days preoperatively was collected. We used CKD Epidemiology Collaboration Equation (CKD-EPI) to calculate the estimated glomerular filtration rate (eGFR) [11].

$$
\begin{aligned}
\mathrm{eGFR}=141 & \times \min (\mathrm{Scr} / \kappa, 1)^{\alpha} \\
& \times \max (\mathrm{Scr} / \kappa, 1)^{-1.209} \\
& \times 0.993^{\text {Age }} \\
& \times 1.018(\text { if female }) \\
& \times 1.159 \text { (ifblack) }
\end{aligned}
$$

Were Scr is the serum creatinine, $\alpha$ is -0.329 for women and -0.411 for men, $\mathrm{k}$ is 0.7 for women and 0.9 for men, min indicates the minimum $\mathrm{Scr} / \mathrm{k}$ or 1 , and max indicates the maximum $\mathrm{Scr} / \mathrm{k}$ or 1 . The pre-existing CKD stages of all patients were determined based on their pre-existing eGFR at the time of unilateral nephrectomy. All the patients were stratified into stages of CKD based on the Kidney Dialysis Outcomes Quality Initiative (K-DOKI) classification, as follows: stage $1, \mathrm{GFR}>90 \mathrm{~mL} / \mathrm{min} / \mathrm{m}^{2}$ with

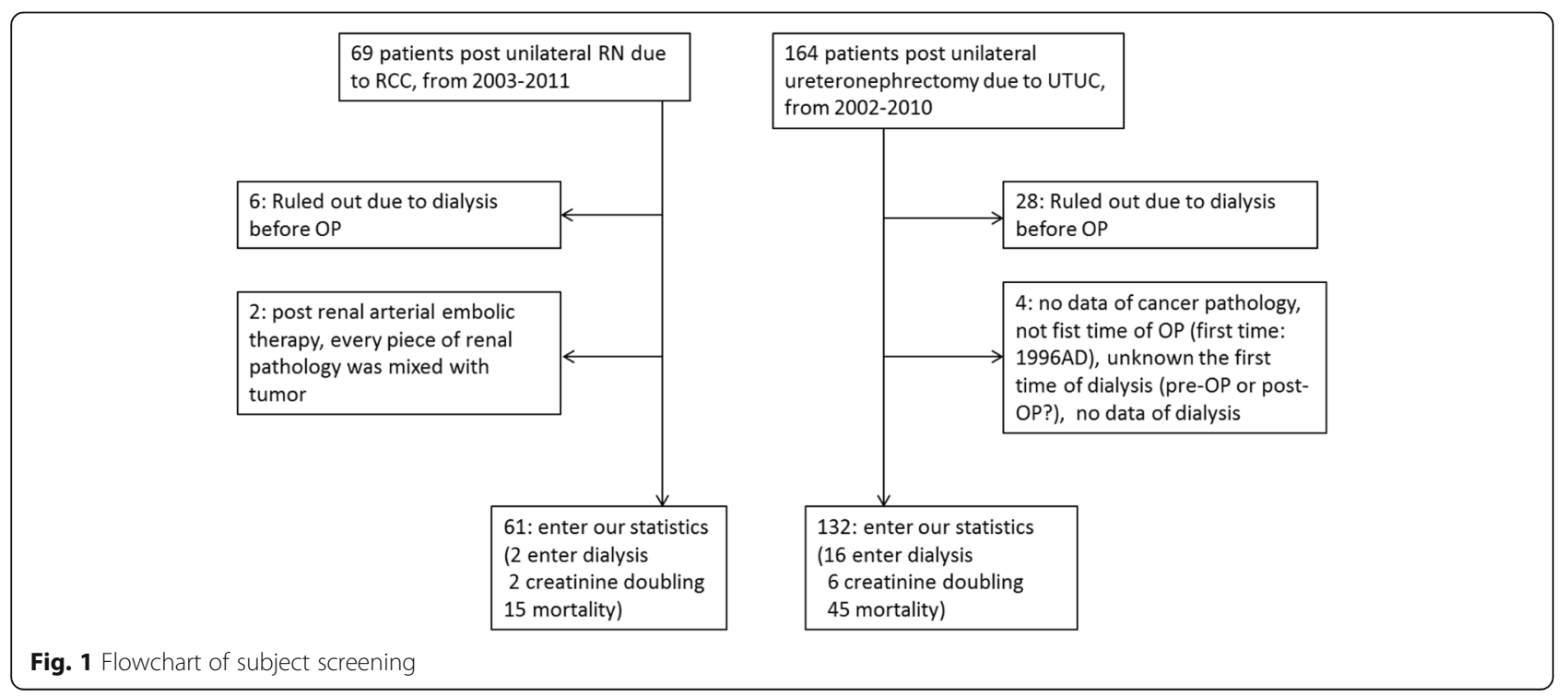


proteinuria or microalbuminuria; stage 2, GFR 60-89 $\mathrm{mL} / \mathrm{min} / \mathrm{m}^{2}$ with proteinuria or microalbuminuria; stage $3 \mathrm{~A}, \mathrm{GFR}=45-59 \mathrm{~mL} / \mathrm{min} / \mathrm{m}^{2}$; stage $3 \mathrm{~B}, \mathrm{GFR}=$ $30-44 \mathrm{~mL} / \mathrm{min} / \mathrm{m}^{2}$; stage 4, GFR $=15-29 \mathrm{~mL} / \mathrm{min} / \mathrm{m}^{2}$; and stage 5, GFR $<15 \mathrm{~mL} / \mathrm{min} / \mathrm{m}^{2}$.

\section{Histochemical staining}

Kidney specimen were thoroughly excision and representation, section are taken from the non-tumorous area, which is at least $1 \mathrm{~cm}$ distance from the tumor. Formalin-fixed paraffin embedded (FFPE) blocks of the non-tumorous kidney parenchyma were retrieved. Tissue sections of $3 \mu \mathrm{m}$ were cut, deparaffinized, and rehydrated. Hematoxylin-eosin (H\&E) stain, periodic acid-Schiff (PAS) stain and Masson trichrome stain were performed as recommended [12].

\section{Pathological evaluation}

The global glomerulosclerosis (GGS) rate and tubulointerstitial (TI) score were semiquantified by 2 nephrologists and one pathologist, who were blinded to the patients' clinical information. For cases with discrepancy, a consensus was made after review the slides together at a multi-headed microscope. The TI score was the sum of the severity level of four pathological features: tubular necrosis (Fig. 2a; 0: normal tubules, 1: rare single necrotic tubule, 2: several clusters of necrotic tubules, and 3: confluence of necrotic clusters), tubular atrophy (Fig. 2b; 0: normal tubules, 1: rare single atrophic tubule, 2: several clusters of atrophic tubules, and 3: confluence of atrophic tubular clusters), lymphocytic infiltrates (Fig. 2c; 0: absent, 1: few scattered cells, 2: group of lymphocytes, 3 : and widespread infiltrates), and interstitial fibrosis (Fig. 2d; 0: absent, 1: minimal fibrosis, 2: moderate fibrosis, and 3: severe fibrosis), ranging from 0 to 12 [13]. GGS rate was the number of glomeruli with global glomerulosclerosis, defined as glomerulus with more than $50 \%$ of area involved by sclerosis, over the number of the glomeruli that can be found in the slides (Fig. 2e). Since GGS developed as an individual getting old, we compared the observed GGS rate with the estimated GGS, calculated by using an equation, (age X 0.5) 10, that proposed by Smith et al. [14]. If the observed GGS rate exceeded the estimated GGS, it was considered as "abnormal GGS rate" (Table 3). For an example, a $40 \%$ of observed GGS in a 80 years old patient (estimated GGS is $80 \times$ $0.5-10=30 \%$ ) was considered abnormal.

\section{Postoperative follow-up}

All the patients were followed by performing cystoscopic examination every 3 months in the first 2 years after nephrectomy, every 6 months in the next 2 years, and annually thereafter. During surveillance, physical examinations and cystoscopic, urine cytological, and periodic imaging studies were performed following institutional guidelines.
Intraluminal recurrence was defined as the recurrence of tumors in the contralateral upper urinary tract or bladder. Metastatic progression was defined as tumor recurrence in the tumor bed or regional lymph nodes and distant metastasis.

\section{End points}

The primary end point was renal outcomes, defined as creatinine doubling or dialysis. The secondary end point was all-cause mortality. If the patients died within 3 months of the primary end point, they were not defined as having the primary end point.

\section{Statistical analysis}

Data were described as mean \pm standard deviation (SD), frequency, or percentage. Student's t test or one-way analysis of variance (ANOVA) was used for comparing the continuous variables between different groups and chi-squared test was used for comparing different distribution of categorical data. Multiple binary logistic regression was applied to explore factors associated with preexisting CKD-EPI and abnormal of GGS rate. Factors associated with TI score were evaluated by multiple linear regressions. We calculated follow-up time as time between the date of unilateral nephrectomy and the date of dialysis or creatinine doubling. The Kaplan-Meier method was used to estimate renal survival rates of the histological GGS normal and abnormal groups and test the difference between these two groups by log-rank test.

Because our patients were more likely to die than to reach renal outcomes, a competitive risk Fin-Gray regression model was used to identify independent associated predictors. All independent variables were included univariable analysis and selected into multivariable analysis under criteria of $p<0.1$. All statistical analyses were performed using SPSS Version 19 (IBM, Armonk, NY, USA) or SAS 9.4 (SAS Institute Inc., Cary, NC,USA), and figures were made using GraphPad Prism 5.0 (GraphPad Software, Inc., California, USA). In all analyses, two-sided $p<0.05$ was considered statistically significant.

\section{Results}

Differences of clinical, laboratory, and pathological characteristics between UTUC and RCC groups

The clinical, laboratory, and pathological characteristics in UTUC and RCC groups are shown in Table 1. Compared to the RCC group, patients of UTUC group were significantly more women, older in age, more use of Chinese herb, and higher percentage of hydronephrosis. There were also significantly more pre-existing CKD, higher creatinine level, lower eGFR, anemic, increased risk of adverse renal outcome after surgery $(p=0.056)$, and higher overall mortality in UTUC group. Both the 




Fig. 2 a Tubular necrosis. The left image shows a typical sample, and the right image shows tubular necrosis (vacuolated cells and sloughed, necrotic cells in tubular lumina, with some tubules lined by a flattened epithelium and some showing frank necrosis). (Periodic acid Schiff staining, $\times 20$ ). b Tubular atrophy. The left image shows a typical sample, and the right image shows tubular atrophy (tubular basement membranes thickening and wrinkling, with simplified tubular epithelial cells, small round tubules with markedly flattened, uniform intratubular casts, and contraction of the tubular lumen adjacent to intact tubules). (Periodic acid Schiff staining, $\times 20$ ). c Interstitial lymphocyte infiltration. Upper figures: The left image shows a typical sample, and the right image shows interstitial lymphocyte infiltration. The renal cortex shows a diffuse interstitial, predominantly mononuclear, inflammatory infiltrate with no changes in the glomerulus. (Periodic acid Schiff staining, $\times 10$ ). Lower figures: The left image shows a typical sample, and the right image shows interstitial lymphocyte infiltration. Tubules in the center of the field are separated by inflammation and edema. (Periodic acid Schiff staining, $\times 20$ s). $\mathbf{d}$ Interstitial fibrosis. The left image shows a typical sample, and the right image shows interstitial fibrosis (connective tissue expansion through tubulointerstitial parenchyma and tubular loss). (Masson's trichrome, $\times 10$ ). e Global glomerulosclerosis score. The left figures show a typical sample, and the right figures shows GGS (a solidified nonretracted glomerular tuft with often recognizable tuft adhesions, splitting of Bowman's capsule, and prominent periglomerular fibrosis). (Upper figures, periodic acid Schiff staining, $\times 20$ and lower figures, H\&E staining)

histopathological TI score and GGS score of kidney remnant shown in Fig. 3 were also significantly higher in the UTUC group. The average GGS rates were $24.12 \pm$ $27.88 \%$ and $10.80 \pm 12.60 \%$ in the UTUC and RCC groups, respectively $(p<0.001)$; and the average TI scores were $4.76 \pm 2.92$ and $2.13 \pm 2.55$ in the UTUC and RCC groups, respectively $(p<0.001)$. Distributions of pre-existing CKD in subjects with UTUC $(N=132)$ and RCC $(N=61)$, as stratified by age and sex, were shown in Fig. 4. The proportion of women in UTUC was higher (men vs women, $43.2 \%$ vs $56.8 \%$ ) but prevalence of pre-existing CKD was slightly higher in UTUC men (80.7\% vs $73.3 \%)$.

\section{Factors associated with pre-existing CKD in UTUC groups}

Since there were patients with renal function impairment before unilateral nephrectomy and was more pronounced in UTUC group, to explore factors associated with pre-existing impaired renal function, results of logistic regression analysis of pre-existing CKD and all the clinical and pathological factors are shown in Table 2 . Tumor type (UTUC), age, hydronephrosis, TI score, and abnormal GGS rate were independently associated with pre-existing CKD. After adjusting all possible confounding factors, presence of UTUC and age became the two factors with significantly higher odd ratio of pre-existing CKD. We further looked into UTUC group, and the pre-existing CKD was likely associated with age, however, presence of abnormal GGS rate became another significant factor $(p=$ 
Table 1 Clinical, laboratory, and pathological characteristics of UTUC and RCC groups

\begin{tabular}{llll}
\hline Variable & UTUC & RCC & $p$ \\
\hline Female & $75(56.8 \%)$ & $15(24.6 \%)$ & $<0.001$ \\
Age (years) & $67.9 \pm 9.5$ & $57.07 \pm 11.58$ & $<0.001$ \\
$\begin{array}{l}\text { Lifestyle } \\
\quad \text { Chinese herb }\end{array}$ & $28(25.0 \%)$ & $4(6.6 \%)$ & 0.003 \\
$\quad$ Smoking & $27(23.5 \%)$ & $16(26.2 \%)$ & 0.686 \\
Comorbidity & & & \\
$\quad$ Hypertension & $50(40.0 \%)$ & $31(51.7 \%)$ & 0.134 \\
$\quad$ Diabetes mellitus & $29(23.2 \%)$ & $16(26.7 \%)$ & 0.607 \\
$\quad$ Hyperlipidemia & $8(6.4 \%)$ & $3(5.0 \%)$ & 0.706 \\
Hydronephrosis & $53(47.3 \%)$ & $2(3.3 \%)$ & $<0.001$ \\
Pre-existing CKD & $101(76.5 \%)$ & $15(24.6 \%)$ & $<0.001$ \\
Renal stone & $14(12.7 \%)$ & $4(6.6 \%)$ & 0.208
\end{tabular}

Laboratory data (Before nephrectomy)

$\begin{array}{llll}\text { BUN }(\mathrm{mg} / \mathrm{dl}) & 21.77 \pm 15.47 & 16.10 \pm 10.94 & 0.015 \\ \text { Crea }(\mathrm{mg} / \mathrm{dl}) & 1.57 \pm 1.30 & 1.14 \pm 0.77 & 0.018 \\ \text { eGFR }\left(\mathrm{ml} / \mathrm{min} / \mathrm{m}^{2}, \text { CKD-EPI) }\right. & 53.8 \pm 24.7 & 80.0 \pm 23.4 & <0.001 \\ \text { GPT }(\mathrm{IU} / \mathrm{L}) & 25.08 \pm 24.84 & 30.79 \pm 26.39 & 0.161 \\ \text { Albumin }(\mathrm{mg} / \mathrm{dl}) & 3.75 \pm 0.55 & 4.04 \pm 0.51 & 0.017 \\ \mathrm{Na}(\mathrm{mEq} / \mathrm{L}) & 137.31 \pm 4.29 & 139.10 \pm 2.53 & 0.021 \\ \text { K (mEq/L) } & 3.96 \pm 0.44 & 3.94 \pm 0.39 & 0.698 \\ \text { WBC }(\times 1000 / \mathrm{ul}) & 7.70 \pm 3.21 & 7.02 \pm 1.91 & 0.126 \\ \mathrm{Hb}(\mathrm{g} / \mathrm{dL}) & 11.60 \pm 2.07 & 13.40 \pm 2.08 & <0.001 \\ \text { PLT }(\times 1000 / \mathrm{ul}) & 218.07 \pm 76.62 & 229.30 \pm 89.14 & 0.398\end{array}$

Renal histopathology

Distribution of TI score

0

$1 \sim 4$

$5 \sim 8$

$\geqq 9$

Distribution of GGS rate

0

$>0 \sim 10$

$>10 \sim 25$

$>25 \sim 50$

$>50$ (DGGS)

Adverse renal outcome

Creatinine doubling

Dialysis

Overall mortality
0.049, Additional file 1: Table S1a) but not TI score. Since GGS could be a consequence of ageing or systemic diseases of hypertension or diabetes, multivariate analysis of logistic regression showed eGFR was the only factor significantly associated with abnormal GGS rate $(p<0.001$, Additional file 1: Table S1b). The non-significances of age, hypertension, and diabetes excluded systemic factors responsible for abnormal GGS rate and suggested the possibility of unexplored mechanisms of GGS in UTUC patients.

Despite of a higher TI score in UTUC group than in RCC group (Table 1), TI score was neither significantly associated with pre-existing CKD in overall patients (Table 2), nor a significant factor to pre-existing CKD and abnormal GGS in UTUC patients (Additional file 1: Table S1a, Table S1b).

\section{Factors associated with pre-existing CKD in RCC groups}

Compared to the significant role of abnormal GGS rate to pre-existing CKD in UTUC group, there were just two cases of abnormal GGS rate in RCC patients. The too few the case numbers resulted in no significance associated with other factors (data not shown).

Though TI score was not a significant factor of pre-existing CKD in all patients as a whole (Table 2), in RCC group the pre-existing CKD significantly associated with TI score and age (Additional file 1: Table S2a, $p=0.010, p=0.003$ ), and TI score further significantly inversed associated with eGFR ( $p=0.015$, Additional file 1: Table S2b).

These observations might suggest that the pathogenesis of kidney function impairment in patients with UTUC and with RCC was different.

\section{Factors associated with the adverse renal progression}

In overall 193 renal cancer patients, 22 of 132 (16.7\%) UTUC patients and 4 of 61 (6.7\%) RCC patients reached the primary end-point developed into ESRD received dialysis or showed creatinine doubling within 5 years after surgery. Competitive survival regression analysis after stepwise selection showed hypertension $(p=0.004)$, pre-existing CKD $(p=0.019)$, or abnormal GGS rate $(p=$ $0.041)$ were risk factors associated with adverse renal outcomes (Table 3).

\section{Discussion}

0.056 The present study demonstrate that UTUC patients had clinical features of female predominant, older in age, significantly higher risks of pre-existing CKD, and pathological findings of abnormal GGS rate and higher TI score than RCC ones. The pre-existing CKD in UTUC patients was associated with older age and abnormal GGS rate, and the abnormal GGS rate was associated with worse pre-existing GFR, but had no relation to age and systemic diseases. On the contrary, pre-existing CKD in RCC patients was associated with older age and

UTUC upper urinary tract urothelial carcinoma, RCC renal carcinoma, CKD chronic kidney disease, GGS global glomerulosclerosis, $T I$ tubulointerstitial, eGFR glomerular filtration rates

Adverse renal outcomes, namely creatinine doubling and entering dialysis Statistics done by unpaired t-test and ${ }^{*}$ Chi-squared test; $p$ less than 0.05 as significant 




Fig. 3 Distributions of global glomerulosclerosis (GGS) rates and tubulointerstitial (TI) scores. (a) GGS rate (b) TI score

higher TI score, and the higher TI score was related to worse pre-existing GFR. Patients with hypertension, pre-existing CKD before unilateral nephrectomy and abnormal GGS rates had a higher risk of creatinine doubling or developing ESRD within 5 years in not only UTUC but RCC patients.

Renal cancer is a common malignancy in countries with high socioeconomic development. Upper urinary tract urothelial carcinoma (UTUC) [2] and renal cell carcinoma (RCC) [1] are the two most commonly seen renal cancers. Although RCC are more common worldwide, especially in western countries, UTUC has higher incidence rate in certain regions, such as Balkan's countries and Taiwan. Nephrectomy with or without ureterecctomy is for treatment of resectable renal cancer. Previous study shows that new-onset CKD developed in patient with T1a RCC after surgery [15]. Similar observation has been disclosed in UTUC patients [7]. In our study, we shows that hypertension, pre-existing CKD, and abnormal GGS rate are indicator for predicting




Table 2 Factors associated with Pre-existing CKD by logistic regression

\begin{tabular}{|c|c|c|c|c|c|c|}
\hline & COR & $95 \% \mathrm{Cl}$ & $p$ value & $\mathrm{AOR}$ & $95 \% \mathrm{Cl}$ & $p$ value \\
\hline \multicolumn{7}{|l|}{ Tumor type } \\
\hline $\mathrm{RCC}$ & 1.00 & Reference & & 1.00 & Reference & \\
\hline UTUC & 9.99 & $(4.92,20.29)$ & $<0.001$ & 3.09 & $(1.18,8.06)$ & 0.021 \\
\hline Gender (male vs female) & 0.65 & $(0.36,1.17)$ & 0.149 & - & - & - \\
\hline Age & 1.14 & $(1.10,1.19)$ & $<0.001$ & 1.12 & $(1.07,1.18)$ & $<0.001$ \\
\hline Chinese herb & 1.39 & $(0.63,3.05)$ & 0.418 & - & - & - \\
\hline Smoking & 0.95 & $(0.47,1.89)$ & 0.878 & - & - & - \\
\hline Hypertension & 1.37 & $(0.75,2.49)$ & 0.304 & - & - & - \\
\hline DM & 1.13 & $(0.57,2.26)$ & 0.727 & - & - & - \\
\hline Hyperlipidemia & 1.18 & $(0.33,4.18)$ & 0.800 & - & - & - \\
\hline Hydronephrosis & 3.46 & $(1.68,7.10)$ & 0.001 & 1.42 & $(0.56,3.63)$ & 0.459 \\
\hline Renal stone & 2.08 & $(0.71,6.12)$ & 0.184 & - & - & - \\
\hline TI score & 1.24 & $(1.12,1.38)$ & $<0.001$ & 1.11 & $(0.96,1.28)$ & 0.153 \\
\hline Abnormal GGS & 4.79 & $(2.18,10.54)$ & $<0.001$ & 2.68 & $(0.99,7.24)$ & 0.052 \\
\hline
\end{tabular}

Abbreviation: OR odd ratio, $\mathrm{Cl}$ confidence interval, $C O R$ crude $\mathrm{OR}, A O R$ adjusted OR. Abbreviations are same as in Table 1

kidney function deterioration after nephrectomy in both RCC and UTUC patients. Thus, it is likely that worse baseline kidney function plays a significant role in promoting CKD development in patient with renal cancer.

In nephrectomy specimens, non-neoplastic kidney disease frequently goes unrecognized [16]. Bijol et al. shows that patients with severe histopathologic findings predict a worse kidney outcome after radical nephrectomy [17]. In our study, we evaluate abnormal GGS rate and TI score in nephrectomy specimens of RCC and UTUC patients. We observed that, in contrast to RCC, UTUC patients had worse TI score and higher GGS rate. Abnormal GGS rate is strongly related to the pre-operation eGFR in UTUC patient, but it is a rare event in RCC patient. However, it is inverse in TI score, where higher TI score is associated with worse pre-operation eGFR in RCC patients but not UTUC. We concluded that the mechanism that lead to renal impairment in these two groups of cancer is likely different. In UTUC, it is the destruction of glomeruli that lead to renal impairment. This process is not related to other clinical

Table 3 Competing risk analysis in the UTUC and RCC groups to develop renal function impairment (creatinine doubling or receiving dialysis)

\begin{tabular}{lll}
\hline Variable & AHR $(95 \% \mathrm{Cl})$ & $p$ \\
\hline Hypertension (yes versus no) & $4.12(1.57,10.84)$ & 0.004 \\
Pre-existing CKD (yes versus no) & $6.20(1.36,28.30)$ & 0.019 \\
Abnormal GGS rate (yes versus no) & $2.41(1.04,5.59)$ & 0.041 \\
\hline
\end{tabular}

Competing risk analysis was used as cause-specific hazard ratio estimation for renal outcomes, whereas death before dialysis or creatinine doubling or within 3 months after dialysis or creatinine doubling was considered as a competing event. Covariates in the multivariate model included tumor type (UTUC vs RCC), age, gender, a history of Chinese herb, smoking, diabetes, hyperlipidemia, hydronephrosis, renal stone, and $\mathrm{Tl}$ score of renal histopathology. Abbreviations are the same as in Table 1 factors, although age may play role in the process. Although tubulointerstitial injury did not show correlation with post-operation kidney function impairment, it is still a frequent event in UTUC. Unlike RCC, where tubulointerstitial injury is likely a consequence of glomerular destruction, this event is not associated with any factors that we look into in UTUC. Aristolochic acid (AA), a compound that is known to cause chronic kidney disease and urothelial carcinoma, has been purposed as a possible cause. Animal model shows that AA nephropathy (AAN) can cause severe tubulointerstitial injuries, including proximal tubular epithelial cell necrosis and transient acute kidney injury [18]. Chen et al. shows that AA related UTUC has higher incidence rate of ESRD than those non-AA related UTUC (28\% vs $12 \%$ ) [19]. However, AA exposure alone is not the sole explanation, because AAN usually shows extensive hypocellular interstitial sclerosis, tubular atrophy, and cellular atypia but spared glomeruli involvement [20]. Besides, we could not determine the incidence of AA intake nor AAN in our patients. The mechanism should be multifactorial and other possible pathogens are yet to be disclosed.

In Taiwan, the prevalent rate of CKD stage $3-5$ is $6.9 \%$ [21]. In our cohort, the prevalent rate of pre-existing CKD in UTUC patients and RCC patients is 76.5 and $24.6 \%$, respectively, and they are significantly higher than the general population in Taiwan. Abnormal GGS rate and TI score also associated with pre-existing CKD, although they are not independent predictors. These findings suggested that factors that accurately reflect the kidney reserved function are yet disclosed. Our study also shows that, in contrast with RCC, the UTUC are more correlated with pre-existing CKD. Although Hung et al. had reported that the aggressiveness of UTUC increased 
with the severity of CKD [22], our finding is the first to show that UTUC is an independent predictor for pre-existing CKD in renal cancer. Based on this observation, it is recommended to thoroughly evaluate the kidney function of patient with UTUC before surgical intervention.

The prevalence of CKD in women and men are variable worldwide [23]. In Taiwan, the incidences of male and female patients with CKD exhibiting renal progression are 11.64 and $12.52 \%$, respectively [24]. In our study, the proportion of women in UTUC was higher (men vs women, $43.2 \%$ vs $56.8 \%$ ) but the prevalence of pre-existing CKD was more commonly seen in men with UTUC (men vs women, $80.7 \%$ vs $73.3 \%$ ). We hypothesize that although men have less likelihood of UTUC development, they are more susceptible to factors that injured the kidney. In animal model, male mice with orchiectomy may inhibit renal injury and female rat prevent renal injury through the expression of vascular endothelial growth factor and endothelial nitric oxide synthase $[25,26]$. Previous studies also disclosed that estrogen has renoprotective effect and testosterone could enhance renal injury [27, 28]. Testosterone and $17 \beta$-estradiol have opposite effects on renal cells in female estrogen receptor knockout mice, as the former could aggravate podocyte apoptosis and glomerulosclerosis but the later inhibited the process [29]. Patients with AAN were usually combined with UTUC [30]. Taking AAN as a model, treatment with $17 \beta$-estradiol in male C57BL/6 mice with AAN reduced serum creatinine levels and attenuated renal proximal tubular injury and renal tubular epithelial cells apoptosis. In the mice kidney tissue and human renal proximal tubule cells (HK-2 cells), $17 \beta$-estradiol attenuated both AA-induced cell apoptosis via inhibiting the p53 signaling pathway [31]. Since most of our female patients are over 55 years old, and are likely menopause, we did not see the renoprotective effect in our cohort. But another recent study from our team revealed that Glycine $\mathrm{N}$-methyltransferase attenuates AAN via decreasing $\mathrm{NAD}(\mathrm{P}) \mathrm{H}$ : quinone oxidoreductase 1 (NQO1) expression in female mouse hepatocytes, and this also implied: First, that male were less tolerant of AA toxicity than female, so acute kidney injury could warn male to stop AA-contained herbs intake [32], and continuous AA-contained herbs intake due to more tolerant of AA toxicity in female, may cause higher incidence of UTUC in Asia, especially in Taiwan [33]. Then, female were expected to have longer lifespan, this also contributed to higher incidence of UTUC in female.

Our study identified that both UTUC and RCC patients after unilateral nephrectomy with abnormal GGS rate, hypertension and pre-existing CKD have higher risk of creatinine doubling or dialysis within 5 years. Under this observation, we found some key points: First, UTUC patients had significantly higher risk of pre-existing
CKD and higher TI scores than RCC ones, but UTUC patients with worse pre-existing GFR (EPI) have higher risk of abnormal GGS rates. Then, Both UTUC and RCC patients after unilateral nephrectomy with abnormal GGS rate have a higher risk of creatinine doubling or dialysis within 5 years, but there was no different risk of creatinine doubling or dialysis within 5 years between UTUC and RCC patients after unilateral nephrectomy. Finally, UTUC and RCC patients with hypertension had a lower 5-year postoperative renal survival. Hwang et al. reported that diabetes mellitus (43.2\%), chronic glomerulonephritis (25.1\%), hypertension (8.3\%), and chronic interstitial nephritis (2.8\%) are major underlying comorbidities of ESRD ${ }^{21}$.

According to our results, the UTUC patients frequently suffered from pre-existing CKD and TI nephropathy than RCC ones, but UTUC ones with worse renal function had higher risk of abnormal GGS rate, and abnormal GGS Rate is histopathological predictor of creatinine doubling or dialysis within 5 years in not only UTUC patients but $\mathrm{RCC}$ ones. Because the renal histopathology of nephrectomized kidneys is a strong predictor, we suggest that pathophysiological analysis of nephrectomized kidneys can be performed to assess the renal outcomes of UTUC patients after unilateral nephrectomy.

\section{Limitation}

The study has several limitations. First, this is a single institute study and selection biased cannot be preventable. A larger multi-institute may be needed to confirm our findings. Then, GGS and TI score are not accurate methods to evaluate chronic kidney injury. The scoring of tubulointerstitial injury is relative subjective, and GGS will be affected by the representativeness of the sampled tissue. Finally, the environmental factors that the patient exposed to are difficult to collect, such as the relationship between the UTUC patients and AA exposure.

\section{Conclusion}

We confirmed the relevance of the renal histopathology of nephrectomized kidneys in predicting the renal survival rate of both UTUC and RCC patients receiving unilateral nephrectomy. Our findings indicated that UTUC patients had significantly higher risk of pre-existing CKD, abnormal GGS rate, and TI score than RCC ones, but UTUC with worse renal function had higher risk of abnormal GGS rate. Patients with hypertension, pre-existing CKD before unilateral nephrectomy and abnormal GGS rate in the renal histopathological analysis of nephrectomized kidneys from unilateral nephrectomy had a higher risk of creatinine doubling or developing ESRD within 5 years in not only UTUC but RCC patients. Thus, we may lend consideration to routine histologic evaluation of the non-tumorous kidney. 


\section{Additional file}

Additional file 1: Table S1a. Factors associated with pre-existing CKD by logistic regression in UTUC group. Table S1b. Logistic regression for Abnormal GGS rate in UTUC group. Table S2a. Factors associated with preexisting CKD by linear regression in RCC group. Table S2b. Factors associated with TI score by logistic regression in RCC group. (DOCX 55 kb)

\begin{abstract}
Abbreviations
AA: Aristolochic acid; AAN: Aristolochic acid nephropathy; ANOVA: One-way analysis of variance; CKD: Chronic kidney disease; CKD-EPI: CKD Epidemiology Collaboration Equation; eGFR: Estimated glomerular filtration rate; ESRD: Endstage renal disease; FFPE: Formalin-fixed paraffin embedded; GGS: Global glomerulosclerosis; H\&E: Hematoxylin-eosin; HK-2: Human renal proximal tubule (cell line); K-DOKl: Kidney Dialysis Outcomes Quality Initiative; NAD(P) $\mathrm{H}$ : Nicotinamide adenine dinucleotide phosphate; $\mathrm{NQO}$ : $\mathrm{NAD}(\mathrm{P}) \mathrm{H}$ : quinone oxidoreductase 1; PAS: Periodic acid-Schiff; RCC: Renal cell carcinoma; SD: Standard deviation; TI: Tubulointerstitial; UC: Urothelial carcinoma; UTUC: UTUC, upper urinary tract urothelial carcinoma
\end{abstract}

\section{Acknowledgements}

We thank the Graduate Institute of Clinical Medicine, Kaohsiung Medical University for supporting this study.

\section{Funding}

The study was supported by Ministry of Science and Technology (NSC 102-2314B-037-012-MY3, MOST 106-2314-B-037-071-MY2); Health and Welfare Surcharge of Tobacco Products, Ministry of Health and Welfare (MOHW103-TD-B-111-05, MOHW104-TDU-B-212-124-003, MOHW105-TDU-B-212-134007); Kaohsiung Medical University Hospital (KMUH103-3 M08, KMUH102-2R09 and KMUH103-3R11); and Kaohsiung Municipal Ta-Tung Hospital (KMTTH-106-014) for supporting this study. The funders played no role in the study design, data collection and analysis, publication decision, or manuscript drafting.

\section{Availability of data and materials}

The datasets used and/or analysed during the current study are available from the corresponding author on reasonable request.

\section{Authors' contributions}

Participated in the conception, analysis and interpretation of data: S-WN, P-IL, M-YL, S-MY, Y-YZ, P-CH, Y-HC, C-CH, I-CK, H-YL, M-CK, W-ML, C-NH, W-JW, LTC, Y-WC and S-JH. Conceived and designed the experiments: S-WN, M-CK, W-JW, L-TC and S-JH. Data collection: S-WN and P-IL. Performed the experiments, contributed reagents/materials/analysis tools and histopathologic analysis: S-WN, S-MY and P-IL. Data analysis: S-WN, M-YL, P-IL, C-CH, Y-HC and S-JH. Wrote and revised the manuscript: S-WN, M-YL, C-CH, P-IL and S$J H$. Revised the figure: S-WN, M-YL, P-CH, P-IL and S-JH. All authors revised it critically for important intellectual content and gave final approval of the version to be published.

\section{Ethics approval and consent to participate}

The study protocol was approved by the institutional review board (IRB) of Kaohsiung medical university (Kaohsiung, Taiwan). The need for consent was waived by the IRB (KMUH-IRB-20120138) and the data was decoded before undergoing analysis. All demographic data and histopathologic data were collected and fulfilled the regulations of this IRB of our hospital.

\section{Consent for publication}

Not applicable.

\section{Competing interests}

The authors declare that they have no competing interests.

\section{Publisher's Note}

Springer Nature remains neutral with regard to jurisdictional claims in published maps and institutional affiliations.

\section{Author details}

'Graduate Institute of Clinical Medicine, College of Medicine, Kaohsiung Medical University, No. 100, Shih-Chuan 1st Road, Kaohsiung 80708, Taiwan. ${ }^{2}$ Division of Nephrology, Department of Internal Medicine, Kaohsiung Medical University Hospital, Kaohsiung Medical University, No. 100, Tzyou 1st Road, 80708 Kaohsiung, Taiwan. ${ }^{3}$ Department of Pathology, Kaohsiung Medical University Hospital, Kaohsiung Medical University, Kaohsiung, Taiwan ${ }^{4}$ Department of Urology, Kaohsiung Medical University Hospital, Kaohsiung Medical University, Kaohsiung, Taiwan. ${ }^{5}$ National Institute of Cancer Research, National Health Research Institutes, Tainan, Taiwan. ${ }^{6}$ Faculty of Medicine, Kaohsiung Medical University, Kaohsiung, Taiwan. ${ }^{7}$ Kaohsiung Municipal Ta-Tung Hospital, Kaohsiung Medical University, Kaohsiung, Taiwan. ${ }^{8}$ Yozen clinic, Kaohsiung Medical University, Kaohsiung, Taiwan. ${ }^{9}$ Master of Public Health Degree Program, College of Public Health, National Taiwan University, Taipei, Taiwan.

Received: 13 March 2018 Accepted: 27 February 2019

Published online: 08 April 2019

References

1. Gandaglia G, Ravi P, Abdollah F, et al. Contemporary incidence and mortality rates of kidney cancer in the United States. Can Urol Assoc J. 2014:8:247-52.

2. Hutchinson R, Haddad A, Sagalowsky A, Margulis V. Upper tract urothelial carcinoma: special considerations. Clin Adv Hematol Oncol. 2016;14:101-9.

3. Suriano F, Brancato T. Nephron-sparing Management of Upper Tract Urothelial Carcinoma. Rev Urol. 2014;16:21-8.

4. Nocks BN, Heney NM, Daly JJ, Perrone TA, Griffin PP, Prout GR Jr. Transitional cell carcinoma of renal pelvis. Urology. 1982;19:472-7.

5. Yang $\mathrm{MH}$, Chen $\mathrm{KK}$, Yen CC, et al. Unusually high incidence of upper urinary tract urothelial carcinoma in Taiwan. Urology. 2002;59:681-7.

6. Stefanovic V, Polenakovic M, Toncheva D. Urothelial carcinoma associated with Balkan endemic nephropathy. A worldwide disease. Pathol Biol (Paris). 2011;59:286-91.

7. Lin MY, Li WM, Huang CN, et al. Dialysis increases the risk of bladder recurrence in patients with upper tract urothelial Cancer: a populationbased study. Ann Surg Oncol. 2018;25(4):1086-93.

8. De Broe ME. Chinese herbs nephropathy and Balkan endemic nephropathy: toward a single entity, aristolochic acid nephropathy. Kidney Int. 2012;81:513-5.

9. Stefanovic V, Radovanovic Z. Balkan endemic nephropathy and associated urothelial cancer. Nat Clin Pract Urol. 2008;5:105-12.

10. Zivcic-Cosic S, Grzetic M, Valencic M, et al. Urothelial cancer in patients with endemic Balkan nephropathy (EN) after renal transplantation. Ren Fail. 2007; 29:861-5.

11. Levey AS, Stevens LA, Schmid $\mathrm{CH}$, et al. A new equation to estimate glomerular filtration rate. Ann Intern Med. 2009;150:604-12.

12. Debelle FD, Nortier JL, De Prez EG, et al. Aristolochic acids induce chronic renal failure with interstitial fibrosis in salt-depleted rats. J Am Soc Nephrol. 2002;13:431-6.

13. Pozdzik AA, Salmon IJ, Debelle FD, et al. Aristolochic acid induces proximal tubule apoptosis and epithelial to mesenchymal transformation. Kidney Int. 2008;73:595-607.

14. Smith SM, Hoy WE, Cobb L. Low incidence of glomerulosclerosis in normal kidneys. Arch Pathol Lab Med. 1989;113:1253-5.

15. Ahn T, Ellis RJ, White VM, et al. Predictors of new-onset chronic kidney disease in patients managed surgically for T1a renal cell carcinoma: an Australian population-based analysis. J Surg Oncol. 2018;117:1597-610.

16. Henriksen KJ, Meehan SM, Chang A. Non-neoplastic renal diseases are often unrecognized in adult tumor nephrectomy specimens: a review of 246 cases. Am J Surg Pathol. 2007;31:1703-8.

17. Bijol V, Mendez GP, Hurwitz S, Rennke HG, Nose V. Evaluation of the nonneoplastic pathology in tumor nephrectomy specimens: predicting the risk of progressive renal failure. Am J Surg Pathol. 2006;30:575-84.

18. de Jonge $H$, Vanrenterghem $Y$. Aristolochic acid: the common culprit of Chinese herbs nephropathy and Balkan endemic nephropathy. Nephrol Dial Transplantat. 2008:23:39-41.

19. Chen $\mathrm{CH}$, Dickman KG, Huang CY, et al. Aristolochic acid-induced upper tract urothelial carcinoma in Taiwan: clinical characteristics and outcomes. Int J Cancer. 2013;133:14-20. 
20. Debelle FD, Vanherweghem JL, Nortier JL. Aristolochic acid nephropathy: a worldwide problem. Kidney Int. 2008;74:158-69.

21. Hwang SJ, Tsai JC, Chen HC. Epidemiology, impact and preventive care of chronic kidney disease in Taiwan. Nephrology (Carlton). 2010;15(Suppl 2):3-9.

22. Hung $\mathrm{PH}$, Shen $\mathrm{CH}$, Chiu YL, et al. The aggressiveness of urinary tract urothelial carcinoma increases with the severity of chronic kidney disease. BJU Int. 2009;104:1471-4.

23. Goldberg I, Krause I. The role of gender in chronic kidney disease. EMJ. 2016;1:58-64.

24. Chang P-Y, Chien L-N, Lin Y-F, Wu M-S, Chiu W-T, Chiou H-Y. Risk factors of gender for renal progression in patients with early chronic kidney disease. Medicine. 2016;95:e4203-e.

25. Kim J, Kil IS, Seok YM, et al. Orchiectomy attenuates post-ischemic oxidative stress and ischemia/reperfusion injury in mice. A role for manganese superoxide dismutase. J Biol Chem. 2006:281:20349-56.

26. Stringer KD, Komers R, Osman SA, Oyama TT, Lindsley JN, Anderson S. Gender hormones and the progression of experimental polycystic kidney disease. Kidney Int. 2005;68:1729-39.

27. Neugarten J. Gender and the progression of renal disease. J Am Soc Nephrol. 2002;13:2807-9.

28. Park KM, Kim Jl, Ahn Y, Bonventre AJ, Bonventre JV. Testosterone is responsible for enhanced susceptibility of males to ischemic renal injury. J Biol Chem. 2004;279:52282-92.

29. Doublier S, Lupia E, Catanuto P, et al. Testosterone and 17beta-estradiol have opposite effects on podocyte apoptosis that precedes glomerulosclerosis in female estrogen receptor knockout mice. Kidney Int. 2011;79:404-13.

30. Yang HY, Chen PC, Wang JD. Chinese herbs containing aristolochic acid associated with renal failure and urothelial carcinoma: a review from epidemiologic observations to causal inference. Biomed Res Int. 2014; 2014:569325.

31. Shi M, Ma L, Zhou L, Fu P. Renal protective effects of 17 beta-estradiol on mice with acute Aristolochic acid nephropathy. Molecules (Basel, Switzerland). 2016;21(10):1391.

32. Chang MM, Lin CN, Fang CC, et al. Glycine N-methyltransferase inhibits aristolochic acid nephropathy by increasing CYP3A44 and decreasing NQO1 expression in female mouse hepatocytes. Sci Rep. 2018:8:6960.

33. Chou YH, Huang $\mathrm{CH}$. Unusual clinical presentation of upper urothelial carcinoma in Taiwan. Cancer. 1999:85:1342-4.

Ready to submit your research? Choose BMC and benefit from:

- fast, convenient online submission

- thorough peer review by experienced researchers in your field

- rapid publication on acceptance

- support for research data, including large and complex data types

- gold Open Access which fosters wider collaboration and increased citations

- maximum visibility for your research: over $100 \mathrm{M}$ website views per year

At $\mathrm{BMC}$, research is always in progress.

Learn more biomedcentral.com/submissions 